Sliabh na mBan bhFionn

Author(s): Peadar Ua Laoghaire

Source: Gadelica: A Journal of Modern Irish Studies, Vol. 1, No. 4 (1913), pp. 209-219

Published by: Gadelica: A Journal of Modern Irish Studies

Stable URL: http://www.jstor.org/stable/30023352

Accessed: 22-06-2016 16:27 UTC

Your use of the JSTOR archive indicates your acceptance of the Terms \& Conditions of Use, available at

http://about.jstor.org/terms

JSTOR is a not-for-profit service that helps scholars, researchers, and students discover, use, and build upon a wide range of content in a trusted

digital archive. We use information technology and tools to increase productivity and facilitate new forms of scholarship. For more information about JSTOR, please contact support@jstor.org.

Gadelica: A Journal of Modern Irish Studies is collaborating with JSTOR to digitize, preserve and extend access to Gadelica: A Journal of Modern Irish Studies 


\section{SLIABH NA MBAN BHFIONN}

Fad $\delta$, nuair a bhí Fionn agus an Fhian i réim i nEirinn, do thuit ní amach i dtaobh an chnuic seo ar a dtugtar Sliabh na mBan. Do tugadh cuid des na mnáibh óga ba bhreághtha a bhí le fághail i nÉirinn an uair sin agus do cuireadh isteach sa chnuc san fé dhraoidheacht iad. Do deineadh sídh-bhrog áluinn istig sa chnuc dóibh, agus do cuireadh isteach sa tsídh-bhrog san chun cómhnuighthe iad. Ba mhar-a-chéile ansan iad agus mná sídhe. Ní feictí iad ach nuair ba mhaith leó jad féin do thaisbeáint. D'fhanadar istig sa chnuc riamh ó shin, agus níor chuadar i n-aois ná i bhfuirbhtheacht le himtheacht aimsire. Taisbeánaid siad iad féin uaireanta, agus an té a gheibhean radharc ar mhnaoi acu ní chuirean sé an radharc as a chuimhne an chuid eile dhá shaoghal. As iad a bheith 'ghá dtaisbeáint féin ar an gcuma san ó am go ham, do tugadh "Sliabh na mBan bhFionn" ar an sliabh. "Sliabh Feimhin" an ainim a bhí roimis sin air.*

Thaisbeánadh cuid des na h-"óg mhná finna" iad féin uaireanta chun tairbhthe dhéanamh; ach ní h-i gcómhnuighe a dheinidís tairbhthe. Uaireanta is díobháil a dheinidís. Dá mbeadh cailín áluinn óg ag éirighe suas sa chómharsanacht, b'fhéidir go dtaisbeánfadh bean acu í féin don chailín sin chun í fhuadach. Do béarfí an cailín chun siubhail isteach i sídh-bhrog

* Deir Seán ó Donabháin ins na nótaíbh atá aige le hAnnálaibh Righeachta Éirean, nách "Sliabh na mBan bhFionn" an ainim a tugadh ar an sliabh san ós na mnáibh breághtha san, ach gur "Sliabh na mBan Feimheann" a tugadh air, i. the Mountain of the Women of Femhin. Nuair a dubhairt o Donabháin an chaint sin, do thaisbeáin sé nár thuig sé ceart na Gaeluinne. Ni féidir "Sliabh na mBan Feimheann" do chur mar Ghaeluinn ar the Mountain of the Women of Femhin. "Sliabh Ban Feimheann" an ceart chuige sin. Tá "Sliabh na mBan Feimheann" chómh holc de Ghaeluinn le "an fear an tighe" mar Ghaeluirn ar the man of the house. Niféidir, i nGaeluinn, rud do dhealú le cás geineamhnach agus é dhealú san am gcéadna le "an." I gcás gur i mbéalaibh na ndaoine atá an ceart, agus gur "Sliabh na mBan bhFionn" an ceart san le rádh.

Is mó dearmhad nách é sin atá déanta ag ó Donabháin ; ach deirimse 6 chroidhe gur chóir iad go léir a mhaitheamh dá mbeadh oiread eile acu déanta aige, mar gheall ar an saothar uasal a dhein sé ar na hAnnálaibh, gan a thuille dá shaothar a bhac.

"Sliabh na mBan bhFionn," dá bhrígh sin, an ainim atá ar an gcnuc san 6 chuir na h-" óg mhná finna " fhéachaint ar na daoine dearmhad a dhéanamb de'n tsean ainim, i. "Sliabh Feimhin." 
an chnuic, agus do fágfí agá muíntir sa bhaile iarlis éigin seana mhná a bheadh 'ghá gcrádh agus 'ghá gciapadh ar feadh tamail, agus ansan do gheóbhadh bás.

Do thárla, tamal mór éigin ó shin, gur deineadh beart de'n tsórd san i n-áit éigin ná raibh a-bhfad ón gcnuc. Bhí leanbh inghine ag duinuasal a bhí 'na chómnuighe san áit. Bhí an leanbh ag déanamh amach ar bheith dhá bhliain déag d'aois, agus bhí sí chómh háluinn, chómh breágh, chómh dathamhail sin, gur bh'ar éigin fhéadadh aoinne a chíodh í a shúile thógaint di, agus aoinne a deireadh aon fhocal ag moladh a breághthachta go gcaitheadh sé seile uirthi, i dtreó go mbeadh fuath ag na daoine maitha dhi agus nár bhaoghal go mbéarfidís leó í. Bhíodh árd fhearg ar an leanbh féin nuair a caithtí na seilí uirthi, agus níor bh'iongna san,--bhíodh cuid acu ná bíodh ró ghlan.

Do mhol seana bhean éigin í lá, agus dhein sí dearmhad de'n tseile chaitheamh uirthi. An lá céadna san do chonaic an leanbh duine des na h-"óg mhná finna" ó'n sliabh. Do buaileadh breóite an leanbh. I gcionn suím laethanta ba léir do gach aoinne nár bh'í féin a bhí sa leabaig i n-aon chor. Gur fuaduigheadh an leanbh, agus gur fágadh iarlis éigin gan mhaith 'na hinead. Tar éis raint aimsire fuair an iarlis bás. Bhí uaigneas agus buairt agus brón ar gach aoinne, agus níor bh'fhiú trácht ar an uaigneas ná ar an mbuairt a bhí ar aoinne seachas an bhuairt a bhí ar athair agus ar mháthair an leinbh. Thuigeadar gur bh'í a leanbh féin, an inghean áluinn a bhí acu agus go raibh a gcroidhe greamuighthe inti, gur bh'í a bhí tar éis bháis. Ach na daoine eólguiseacha tuisgionacha a bhí ann, bhí fhios acu go maith gur bh'í an iarlis a bhí tar éis bháis, agus gur bh'amhlaidh a fuaduigheadh an inghean álujnn.

Bhí bean 'na cómhnuighe ag bun an tsléibhe, ar an dtaobh theas. Bean abhrais a b'eadh í. Do curtí olan chúichi isteach ó'n dtír mór-thímpal, agus dheineadh sí an olan do chíoradh agus do ghlanadh agus do shlámadh agus do shníomh; agus nuair a bhíodh an snáth tochraiste aici 'na cheirtlíníbh deasa cruinne cruadha, chuireadh sí abhaile é ag trial ar an muíntir go mba leó é, agus do curtí ag trial ar fhigheadóir é, agus deintí bréid de. Ansan do dheineadh an táiliúir casóg de d'fhear an tighe, nú cóta mór, nú do dheineadh bean an tighe clóca dhi féin de; agus gach aoinne a chíodh an chasóg nua ar an bhfear, nú an clóca ar a mhnaoi, deiridís: "Go mairir agus go gcaithir é!" 
Uaireanta bhíodh ní ba mhó de'n olan ag an mnaoi abhrais 'ná mar fhéadadh sí a chíoradh agus a shlámadh agus do shniomh i gcaitheamh an lae, agus b'fhéidir an mhuíntir go mba leó é 'ghá fhiafruighe cad fé ndeár an righneas. Ansan thugadh an bhean abhrais tamal de'n oidhche ag obair agus solus árneáin aici, a d'iarraidh na hoibre dhéanamh agus a d'iaraidh an righnis do luigheadú. Uaireanta, nuair a bhíodh mórán aici le déanamh agus an ghlaodhach ró dhian air, d'fhanadh sí cuid mhaith de'n oidhche ag árneán.

Oidhche dá raibh sí ag árneán ar an gcuma san agus an saoghal 'na gcodla, d'airigh sí mar bheadh daoine ag teacht chun an doruis chúichi. D'osgail an dorus, agus do bhuail chúichi an dorus isteach mórsheisear ban agus ualach éigin idir a lámhaibh acu. Nuair fhéach sí cruinn ortha féin agus ar an ualach, chonaic sí gur bean mharbh, nú bean a bhí i laige, a bhí acu á thabhairt leó eatortha. Thugadar isteach an bhean, agus shíneadar ar an úrlár í. Do phreab an bhean abhrais 'na suidhe, agus chaith sí uaithi an obair. "An marbh atá an bhean san ?" ar sisi leó.

"Ní headh," arsa duine acu ; " níl ach iaracht de laige uirthi."

Do phreab an bhean abhrais agus thug sí léi saghas éigin leighseana a bhí istig aici. Chuireadar an bhean a bhí i laigechuireadar i n-aice na teine í; agus pé dochtúireacht a dheín an bhean abhrais uirthi níor bh'fhada go dtáinigh sí chúichi féin. Do tháinig sí chúichi féin i dtreó gur éirigh sí aniar ar a cabhail. D'ól sí deoch ó'n mnaoi abhrais, agus d'ith sí raint bídh uaithi, ach níor labhair sí focal amach as a béal. Do labhair an bhean abhrais léi go minic an fhaid a bhí sí ag tabhairt an bhídh di, ach níor tugadh aon fhreagra uirthi. Nuair a bhí sí tagaithe chúichi féin ar fad, agus a hanál aici dá fhághail go breágh bog, dubhairt an bhean abhrais léi mar seo: "Sín anso go fóil, a 'nínó, ar an leabaig, agus tiocfidh do neart duit." Do shín. Ansan dubhairt duine de'n mhórsheisear leis an mnaoi abhrais :

“A bhean an bhréidín, má's bréidín seo ar siubhal agat, Cíoram é, slámam é ; ach is feárde sinn congnamh fhághail."

Le n-a línn sin do rug sí ar chuid de'n olan, agus dhírigh sí ar an olan do chíora agus do shlámadh. Le línn an fhocail a rádh dhi "Is feárde sinn congnamh fhághail," d'fhéach sí ar mhnaoi eile den mhóirsheisear. Do labhair an bhean san an chaint chéadna, i.:

A bhean an bhréidín, má's bréidín seo ar siubhal agat, Cióram é, slámam é ; ach is feárde sinn congnamh fhághail. 
D'fhéach sise ar an gcuma gcéadna ar an dtrímhadh bean. Dubhairt an trímhadh bean an dán céadna, i. :

A bhean an bhréidín, más bréidin seo ar siubhal agat,

Cíoram é, slámam é ; ach is feárde sinn congnamh fhághail,

agus d'fhéach sí ar an gceathramhadh bean, agus dubhairt an ceathramhadh bean an chaint chéadna. Mar sin dóibh go dtí go raibh an mhórsheisear acu ar a ndíthal, beirt acu ag cíoradh, agus beirt acu ag slámadh, agus duine acu ag sníomh, agus beirt acu ag tochrais agus iad ag obair ar seól.

Bhí an bhean abhrais ag féachaint ortha, agus chonaic sí an obair áluinn a bhí acu á dhéanamh, agus bhí áthas uirthi. Chonaic sí an $t$-ualach mór oibre a bhí roimpi le déanamh, chonaic sí ag dul i luighead é go tiugh, agus na ceirtlíní deasa ag dul i líonmhaireacht, agus bhí áthas mór uirthi. Chonaic sí, leis, go raibh an obair á dhéanamh ní b'fheár ná mar fhéadfadh sí féin é dhéanamh, agus do chuir san iongna uirthi i dteanta an áthais. Bhí an cíoradh ní b'fhéar, mar dheineadh sé an olan ní ba bhoige. Bhí an slámadh ní b'fheár, mar dheineadh sé an olan ní ba réidhe. Bhí an sníomh ní b'fheár, mar do dheineadh sé an snáth do réir a chéile ar aon raimhdeas, gan oiread agus aon chasadh amháin ann ní ba mhó ná ní ba lugha ná mar ba cheart, gan sgruig ${ }^{1}$ ná snuidhm air, gan caolú ná ramhrú air, ach é go breágh, sleamhain cómhchaol cómhthéagartha. Bhí áthas mór uirthi agus í ag féachaint ar an obair, mar bhí fhios aici cad é a fheabhas a thaithnfadh an snáth san leis an muíntir go mba leó é, nuair a chífidís é.

Nuair a bhí sí tamal ag féachaint ortha ar an gcuma san, tháinig míogarnach uirthi, agus thuit a codla uirthi go sámh.

Nuair a dhúisigh sí as a codla, bhí sé 'na lá gheal. Chruinnigh sí a meabhair, agus d'fhéach sí 'na tímpal. Ní raibh duine ná daonaidhe sa tig ach í féin. Bhí an mhórsheisear imighthe. D'fhéach sí i dtreó na leapan. Ní raibh aoinne sa leabaidh. D'fhéach sí i dtreó na háite 'nar cheart an chruach mhór olla bheith ann. Ní raibh aon phioc de'n olan ann, ach bhí cruach bhréagh mhór cheirtlíní i n-inead na holla. Ansan do thuig sí gur dhein an mhórsheisear ban an obair go léir, agus nuair a bhí an obair déanta acu gur imthighdar agus isi 'na codla. Ag

\footnotetext{
1 sgruig, i. áit sa tsnáth a bhíon níos caoile ná an chuid eile agus go dtéidhean an casadh ann níos cruadh ná mar a théidhean sé sa chuid eile.
} 
machtnamh di ansan ortha, agus ghá dtabhairt chun a cuimhinte do thuig sí 'na haigne ná feaca sí riamh 'na súilibh cinn mná ba bhreághtha ná iad; ach b'í an t-ochtmhadh bean, i. an bhean a cuireadh sa leabaig, an bhean ba bhreághtha dhíobh go léir. D'fhéachadar go léir, i. an mhórsheisear, breágh thar na beartaibh go dtí gur chuimhnigh sí i gceart ar an mnaoi a thug sí as an laige. Mná breághtha, mná fíor bhreághtha, a b'eadh an mhórsheisear, dá mba ná beadh ann ach iad. Ach i n-aice na mná a bhí sa leabaig mná grána a b'eadh iad.

Ach cé'r bh'í an bhean áluinn óg a bhí sa leabaig ? Agus cad fé ndeara don lagachar úd teacht uirthi ? Agus cad fé ndeara dhi gan aon fhocal do labhairt? Nú cad a thug ann i n-aon chor í ? Níor dheabhruigh sí gur bhain sí leis an gcuid eile acu. Ba dhóich le duine ortha gur bh'amhlaidh a fuaradar lasmuich i n-áit éigin í, agus í sa bhfanntais, agus gur thugadar leó isteach í chun í thabhairt as an bhfanntais. Sin mar a bhí an bhean abhrais ag machtnamh ar an sgéal, agus é ag teip uirthi tón ná ceann fhághail air. B'éigion di éirighe as.

Do thárla, raint aimsire 'na dhiagh san, go raibh ualach mór olla aici airís le cíoradh agus le slámadh agus le sníomh agus le tochrais, go raibh eagal' uirthi ná tiocfadh léi go deó na habhraisí bheith olamh i n-am aici don mhuíntir a chuir chúichi iad. Bhí lá ó mhaidin caithte aici ag déanamh na hoibre, agus bhí éacht de'n obair gan déanamh agus an oidhche ag tuitim. Thug sí léi solus árneáin, agus shocaruigh sí ar an oidhche thabhairt ag obair. Do las sí an solus, agus chrom sí ar an obair. Ní raibh sí a-bhfad ag obair nuair a hosgaladh an dorus, agus bhuail chúichi isteach bean agus caipín a clóca amach ar a ceann aici. Dhein sí suas ar an áit 'na raibh an bhean abhrais ag obair, agus sháidh sí a dhá láimh san olan, agus

A bhean an bhréidín, más bréidín seo ar siubhal agat,
Cíoram é, slámam é; is feárde sinn congnamh fhághail,

ar sisi. Ní raibh ach an dán beag ráidhte aici nuair a bhuail an tarna bean isteach, agus sháidh sí an dá láimh sa n-olan, agus dubhairt sí an leath-rann céadna. Níor bh'fhada go raibh an mhórsheisear acu istig, agus na lámha sáidhte san olan acu, agus iad ag obair go tiugh. Bhí fhios ag an mnaoi abhrais go maith cé'r bh'iad a bhí aici, agus bhí áthas mór uirthi, mar bhí fhios aici nár bh'fhada go mbeadh an obair go léir déanta, agus déanta go maith. 
Chomáineadar leó ag obair, go dtí go raibh an tslamairc ${ }^{1}$ dheirineach den olan 'na snáth, agus an snáth deirineach tochraiste ar an gceirthlín deirineach, agus an cheirthlín sin caithte ar an gcruaich ceirthliní a bhí fásta sa chúinne. "Táim ana bhuidheach díbh, a uaisle," ar san bhean abhrais ; " ní fios cathin a bheadh an méid sin oibre déanta agam dá mbeadh orm é dhéanamh am aonar. Táim ana bhuidheach díbh, a uaisle!"

"Is ceart cómhar do dhíol, a bhean mhacánta," arsan chéad bhean a tháinig isteach. "Dheinis-se dhúinne an oidhche eile úd a bhíomair anso ní ná raibh ar ár gcumas féin a dhéanamh. Isé is lugha is gann dúinne teacht agus an congnamh so thabhairt duitse. Ní fios ná go mb'fhéidir go mbeadh gá againn led chabhair airís."

"Agus isé is lugha is gann dómhsa cabhair a thabhairt uaim pé uair a bheidh gá leis an gcabhair," arsan bhean abhrais.

D'éirighdar go léir, agus tharaigeadar caipíní a gclócaí anuas ar a gceanachaibh, agus d'imthighdar.

Do deineadh an obair chómh maith san gur tugadh fé ndeara ar fuaid na cómharsanachta feabhas na hoibre, agus gur mhéaduig ar an gcúram a curtí ar an mnaoi abhrais sin. Thagadh an olan 'na beartaibh móra troma ag trial uirthi. Nuair a bhíodh an iomad den obair aici le déanamh, agus gan ar a cumas teacht air, pé díthal a dhéanfadh sí ná pé árneán a dhéanfadh sí, thagadh an mhórsheisear agus dheinidís an obair di.

Fé dheire tháinig an t-am 'na raibh an leanbh úd adubhradh, go raibh sí ag dul chun báis, agus an bhuairt go léir ar a hathair agus ar a máthair. Ansan tháinig an $t$-am 'na raibh daoine tuisgeanacha ghá rádh nár bh'í an leanbh féin a bhí ann i n-aon chor; gur fuaduigheadh an leanbh féin, agus ná raibh sa leabaig ansúd ach iarlis a fágadh ann nuair a fuaduigheadh an leanbh. Ansan tháinig an t-am agus fuair an iarlis bás.

An oidhche chéadna a fuair an iarlis bás, tháinig an mhórsheisear isteach go dtí an bhean abhrais agus cailín acu agus iad á hiompar eatortha, díreach mar a bhí an chéad uair úd.

" Tá gá led chabhair againn, a bhean mhacánta," arsan chéad bhean díobh a labhradh i gcómhnuighe.

Do phreab an bhean abhrais chúcha, agus thóg sí ar a baclainn an cailín a bhí, dar léi, marbh nú i bhfantais chómh trom san gur

1 slamairc olla, i. an lán duirnn de'n olan a tógtar chun é shlámadh (i. chun é tharac agus do chimilt go dtí go mbeadh sé breágh bog réidh). 
cuma é nú í bheith marbh gan méam inti. Ní túisge fhéach sí uirthi ná mar aithin sí í. An leanbh a fuaduigheadh isí a bhí ann. Ní baoghal gur leog an bhean abhrais uirthi gur aithin sí í. Thóg sí léi síos sa tseómra í agus shín sí ar a leabaig féin í. Chrom sí ar bheith a d'iaraig í thabhairt as an bhfanntais díreach mar a dhein sí leis an gcéad chailín úd. Bhí rud aici go dtugtí biorán suain ${ }^{1}$ air. An té go sádhfí an biorán suain sin 'na cheann, thiocfadh codla air a bheadh ana chosmhail le bás, agus ní thiocfadh sé as an gcodla san go dtí go dtaraiceófí an biorán suain as a cheann. Do sháidh an bhean abhrais an biorán suain $\mathrm{i}$ gceann an chailín, ach dhein sí é a ganfhios don mhórsheisear. Bhí an mhórsheisear ag cíoradh agus ag slámadh agus ag sníomh, agus bhí an bhean abhrais a d'iaraidh an chailín do thabhairt as an bhfanntais, dar léi, agus bhí an oidhche ag imtheacht.

Fé dheire bhí an lá ag teacht, agus ní raibh an cailín ag teacht chúichi féin ná aon phioc dá dheabhramh uirthi go dtiocfadh sí chúichi féin go luath. Bhí an mhórsheisear ag cogarnuig go dian. I ndeire na cogarnaighe do labhair an chéad bhean acu do labhradh i gcómhnuighe.

" A bhean an tighe," ar sisi, " ní foláir dúinne bheith ag imtheacht. Tabhair-se aire mhaith don chailín sin, agus tiocfimíd anso chughat airís nuair a thiocfidh an oidhche. Beidh sí tagaithe as an bhfantais um an dtaca san. Tabhair aire mhaith dhi, agus tabharfar do thuarasdal go maith dhuit." "Déanfad, a uaisle," arsan bhean abhrais. Ansan d'imthighdar.

Tháinig an lá. Chómh luath agus bhí sé 'na lá gheal do tharaig an bhean abhrais an biorán suain a ceann an chailín. Tháinig a meabhair don chailín láithreach, agus a ciall, agus d'éirigh sí. D'aithin sí an bhean abhrais. D'inis sí don mhnaoi abhrais conus mar a tugadh deoch éigin di a bhain a meabhair di agus ná feidir sí cá raibh sí as san amach, go dtí go dtáinig a meabhair di agus í ar a leabaig ag an mnaoi abhrais.

Thug an bhean abhrais rud le $n$-ithe agus le $n$-ól di. Ansan, chómh luath agus bhí an bia agus an deoch caithte aici, agus i láidir a dóthin chun gluaiste, chuir sí clóca léi féin uirthi, agus chuir sí caipín an chlóca amach ar a ceann, i dtreó ná féadfadh aoinne a haghaidh a dh'fheisgint. Ansan do bhuail an bheirt amach agus

1 Níor airigheas an focal biován suain riamh go dtí gur airigheas sa sgéal so é. D'airighinn an focal braon suain : is dócha gur bh'ionan é sin agus sleeping draught. B'fhéidir gur bh'ionan 'biorán suain' agus an tsnáthad le n-a dtugtar morphia do dhuine. 
thánadar go tig muíntire an chailín. D'inis an bhean abhrais do'n athair agus do'n mháthair an sgéal go léir tríd síos. Thuigeadar é go maith. Bhí áthas ana mhór ortha, ní nár bh'iongna. Bhíodar ana bhuidheach de'n mhnaoi abhrais, agus dubhradar léi airís agus airís eile go ndéanfidís an bheart a bhí déanta aici dhóibh do chúiteamh léi.

Tháinig sí a-bhaile, agus bhí sí ag cur agus ag cúiteamh 'na haigne, féachaint cad é an freagra thabharfadh sí ar na mnáibh uaisle úd nuair a thiocfidís. Níor mheas sí go raibh aon fhreagra a b'fheár a thabhairt ortha ná a rádh leó go dtáinig an cailín chúichi féin as an bhfanntais agus gur imthigh sí. Nuair a tháinig an oidhche bhí sí ag faire chúichi agus ag feitheamh. D'imthig tusach na hoidhche, agus níor thánadar. Bhí lár na hoidhche ann agus iad gan teacht. Thuit a codla uirthi ar an dtínteán, ach níor thánadar. Tháinig an lá agus níor thánadar. Tháinig an oidhche airís agus níor thánadar. D'imthig lá, agus dhá lá, agus seachtmhain, agus mí, agus níor thánadar 'na goire. D'imthig an bhliain. Dubhairt sí lé féin ná tiocfidís a thuile.

Bhí dhá bhliain imighthe. Bhí sí ag obair, agus ana chruach olla aici le cíora agus le slámadh agus le sníomh. Bhí an oidhche tar éis tuitim, agus bhí an choinneal árneáin ar lasadh aici, agus í ghá socarú féin chun na hoidhche chaitheamh ag obair. Do tógadh an laiste agus do hosgaladh an dorus, agus bhuail chúichi isteach an chéad bhean úd, agus caipín a clóca ar a ceann aici chómh fada san amach nár fhéad an bhean abhrais a dh'fheisgint ach an dá shúil,-ach bhí an dá shúil sin ar lasadh go géar agus go haibig. Dhein sí suas ar an olan agus sháidh sí a dhá láimh ann, agus dubhairt sí :

A bhean an bhréidín, más bréidín seo ar siubhal agat, Cíoram é, slámam é; is feárde sinn congnamh fhághail.

Is ar éigin a bhí an focal deirineach ráidhte aici nuair siúd isteach an tarna bean agus caipín a clóca amach ar a ceann aici agus a dhá súil ar dearg lasadh fén gcaipín. Siúd chun na holla í, agus sháidh sí a dhá láimh ann, agus dubhairt sí an chaint chéadna. Bhíodar ag teacht ar an gcuma san go dtí go raibh trí naonúir acu istig agus iad ag obair go dian.

Bhí sgannra ag teacht ar an mnaoi abhrais, ach níor leog sí uirthi go raibh. Bhí fhios aici go raibh droch fhuadar éigin fútha, agus bhí sí ag faire chúichi. Fé dheire do labhair bean acu, an chéad bhean úd do labhradh i gcómhnuighe. 
"Eirig, a bhean an tighe," ar sisi, " agus cuir síos tuile teine dhúinn. Tá an oidhche fuar."

Ní raibh an oidhche fuar. Agus bhí teine mhaith sa tínteán cheana. Ach d'éirigh sí agus chuir sí tuile móna ar an dteine. Do leog sí uirthi go raibh áthas ana mhór uirthi mar gheall ar an obair a bheith acu á dhéanamh chómh tiugh. "Cuir an corcán mór san thall ar an dteine, a bhean an tighe," arsan bhean a labhradh; "tá tart agus ocras ag teacht orainn." Corcán ana mhór a b'eadh é. D'fhéadfí duine do chur isteach ann agus é bheiriú 'na bheathaig ann. Nuair a bhí an corcán ar an dteine do labhair an bhean airís.

"Imthig," ar sisi, " agus tabhair leat uisge, agus cuir sa chorcán san é, nú brisfar é. Tá an teine ró theith."

Thug an bhean abhrais fé ndeara iad go léir ag bagairt ar a chéile agus ag gáirí fé $\mathrm{n}$-a $\mathrm{n}$-anál. Níor leog sí aon ní uirthi. Do thóg sí crúsga léi, agus chuaidh sí amach chun an tobair, agus thug sí crúsga uisge isteach léi, agus chaith sí an t-uisge isteach sa chorcán. Ní líonfadh fiche crúsga an corcán. Do leog sí uirthi ana dhithneas a bheith uirthi ag ruith chun an tobair agus ag teacht, a d'iaraidh an chorcáin do líonadh chómh luath agus do b'fhéidir é. Nuair chonacadar ag déanamh an dithinis í, bhí anabhagairt acu ar a chéile agus ana gháirí ar siubhal acu fé $n$-a n-anál. Ní raibh aon choinne acu go raibh aon droch amhras aici ortha.

Nuair a bhí raint mhaith des na crúsgaíbh uisge tabhartha léi aici ó'n dtobar, do rith sí amach chun ceann eile thabhairt léi agus dubhairt sí, ag gabháil amach di: "Ní mór dhom breis dithinis a dhéanamh, a uaisle, nú beidh sibh marbh ag an dtart agus ag an ocaras sar a mbeidh an corcán san lán agam." Bhí sí ag ruith ag gabháil amach di, agus do lean sí ag ruith go dtí go raibh sí ró fhada ón ndoras chun iad a dh'aireachtain fothraim a cos. Ansan do stad sí, agus chaith sí dhi a bróga, agus tháinig sí thar $\mathrm{n}$-ais gan aon fhothram a dhéanamh. Nuair a bhí sí $\mathrm{i}$ n-aice an doruis, i leathtaoibh, chuir sí cluas uirthi féin féachaint an aireóch sí iad ag déanamh aon chainte. D'airig. "Ní fada go mbeidh sé lán a dhóthin," arsan bhean a labhradh i gcómhnuighe.

"Cad a dhéanfimíd ansan, a ríogan ?" arsa bean eile acu.

"Cuirfimíd isteach ann í, agus beireóimíd 'na beathaig í. Cuirfimíd biorán suain inti ná bainfar aisti go ceann tamail," arsan chéad bhean.

Nuair airig an bhean abhrais an focal san, do shleamhnuigh sí thar n-ais chun na háite 'nar fhág sí an crúsga agus na bróga. 
Chuir sí uímpi na bróga, agus do rith sí tímpal go dí an taobh eile de'n tig. Ansan d'osgail sí a béal agus a cliabh, agus chuir sí liú aisti go hárd agus go bínn, liú a hairigheadh breis agus míle mórthímpal ón áit 'na raibh sí 'na seasamh. "A chómharsain a pú-ú-ú-ú-ú-ú!” ar sisi, “ ruithig, ruithig, ruithig! Tá Sliabh na mBan bhFionn trí theine! Sliabh na mBan bhFionn trí theine !! Sliabh na mBan bhFionn trí theine !!!"

D'airig na trí naonúir a bhí istig an liú agus an ghlaodh agus an fógradh. Chaitheadar an obair as a lámhaibh, agus siúd amach iad, agus iad ag baint an doruis dá chéile, agus siúd suas an cnuc iad chómh mear agus bhí sé 'na gcosaibh. Chaith an bhean abhrais ar an dtalamh i féin go dtí go rabhadar imighthe ón ndorus. Ansan do rith sí isteach, agus dhún sí an dorus, agus chas sí an eochair sa ghlas, agus chuir sí geasa ar an eochair an dorus a chimeád dúnta. Chuir sí an ursal 'na seasamh ag an iarta, agus chuir sí de gheasaibh uirthi gan coruighe as an áit sin. Bhuail sí buille de'n tuaig i mbloc adhmaid, agus chuir sí de gheasaibh ar an dtuaig gan coruighe as an áit sin. Chuir sí gach aon rud eile in' áit féin ar an gcuma san, agus chuir sí iad go léir fé gheasaibh cruadha gan coruighe as a n-áiteanaibh go dtí go bhfuasgalóch sí féin iad ós na geasaibh. Ar éigin a bhí an rud deirineach curtha daingean fés na geasaibh aici, nuair airigh sí na mná uaisle ag teacht chun an doruis. Thug duine acu iaracht ar an laiste dh'árdú. Ní raibh aon mhaith ann. Bhí an glas ar an ndorus. "Osgail an dorus, a bhean an bhréidín," arsan bhean amuich, "go gcríochnóimíd an obair duit."

" Ní osgalód," ar sisi, " mar do curfí sa chorcán me!"

"Osgail, osgail, a eochair an ghlais!" arsan bhean amuich.

" Ní fhéadfinn é," arsan eochair. "Táim anso sáidhte sa ghlas, agus táim fé gheasaibh cruadha an dorus a chimeád dúnta."

"Osgail, osgail, a ursal na lorgan bhfada!" arsan bhean amuich.

"Ní fhéadfinn é," arsan ursal. "Táim anso am sheasamh am áit féin i n-aice na teine, agus mo cheann leis an iarta, agus na geasa cruadha orm gan coruighe as an áit seo."

" Osgail, osgail, a thuagh!" arsan bhean amuich.

"Ní fhéadfainn é," arsan tuagh. "Táim anso am áit féin, agus mo bhéal sáidhte san adhmad, agus na geasa cruadha orm gan coruighe as an áit 'na bhfuilim.

" Osgail, osgail, a cheirtlín!" arsan bhean amuich.

"Ní fhéadfinn é," arsan cheirtlín. "Táim anso san áit 'nar fhágais me, agus na geasa cruadha orm gan coruighe as go dtógfar díom na geasa úd a chuiris féin orm nuair a chaithis uait me." 
"Osgail, osgail, a roth an turainn!" arsan bhean amuich.

"Ní fhéadfinn é," arsa roth an turainn. "Tá an tsrang orm, agus ní féidir dom coruighe gan cead ón sraíng."

"Osgail, osgail, a shrang!" arsan bhean amuich.

"Ní fhéadfinn é," arsan tsrang. "Táim ar an roth, agus ní féidir dom a dhéanamh ach an tromán do chasadh."

"Osgail, osgail, a thromán!" arsan bhean amuich.

"Ní fhéadfinn é," arsan tromán. "Tá na geasa cruadha orm gan a dhéanamh ach an fhearsad so do chasadh."

Chomáineadar leó ar an gcuma san ag glaodhach ar na neithibh a bhí istig, agus á iaraidh ar gach ní dhíobh an dorus a dh'osgailt dóibh, ach do theip gach aon rud ortha, mar bhí gach aon rud fé gheasaibh in' áit féin. Fé dheire chuimhnighdar ar rud nár cuireadh fé gheasaibh riamh, agus nár bh'fhéidir a chur fé gheasaibh an fhaid a fágfí istig é, mar ní raibh aon ghnó le déanamh istig aige. Ach bhí an bhean abhrais ró ghasta dhóibh. Bhí fhios aici ná féadfadh sí aon gheasa do chur ar rud ná raibh aon tairbhthe aige le déanamh. B'é rud é ná uisge na gcos. Nuair nár bh'fhéidir na geasa do chur air, isé rud a dhein sí leis ná é chaitheamh an dorus amach sar ar dhún sí an dorus.

"Osgail, osgail, a uisge na gcos!" arsan bhean amuich.

" Ní fhéadfinn é," arsa uisge na gcos. "Táim anso féd' chosaibh san aoileach."

Nuair a fuaradar an freagra san ó uisge na gcos, bhí fhios acu go raibh buaidhte ortha. D'imthighdar go feargach. Táid siad thuas i n-áit éigin sa chnuc ó shin, agus níor airigheas gur thánadar anuas fós.

O Mháire Ruadh, máthair Pheig, iseadh airigheas an sgéal san. (Isí Peig Mháire Ruaidh a dh'inis 'Séadna ' dhom). Bhí a lán eile sa sgéal, ach tá sé imighthe as mo cheann, mar bhíos ró óg nuair airigheas é.

PEADAR UA LAOGHAIRE. 